\title{
Research on Computer Graphics Processing Technology Innovation in the Big Data Era
}

\author{
Bing Wang ${ }^{1, \text { a }}$ \\ ${ }^{1}$ Maritime Department of Henan Communication Vocational Technology College, Zhengzhou, \\ Henan, China, 450005 \\ a email
}

Keywords: Computer Graphics Processing Technology, Innovation, Big Data Era

\begin{abstract}
With the continuous development of computer technology and network technology, there has been massive data information, the world has moved from the era of data to the era of big data. The arrival of the era of big data to the computer information processing technology has brought a very big impact on the existing computer information processing technology is difficult to meet the massive data processing needs, at the same time easy access to massive data, it also brings a certain amount of data security issues. Article on the concept of computer graphics information processing technology and big data defines, and then analyzes the opportunities and challenges for the next era of big data computer graphics information processing technology facing Finally, the development direction of the next big data era of computer graphics information processing technology will be discussed.
\end{abstract}

\section{Introduction}

In recent years, with the growing popularity of proposed computer technology and network technology, DD, micro-blogging, letter, e-commerce, social networking, networking and other concepts and implementation is largely changed people's daily life, but also with to the huge amounts of data, people entered the era of big data. In the era of big data, generating vast amounts of information every day data, and this massive data continue to accumulate over time, conventional computer information processing technology has been difficult to meet the massive data processing needs, which brought to the computer information processing technology huge new challenges, people need new ideas and innovative perspective to deal with big data problems. In addition, the rapid development of network technology today, the vast amounts of data also makes anyone can access information from anywhere, anytime they want, which will inevitably involve data security issues.

Computer graphics technology computer network system as a platform, combined with each other to achieve the people's subjective sense of real images and graphics between a variety of computer graphics, image processing software, people's subjective handling and operation provides a lot of convenient, with the rapid development of modern science and technology, applications of computer graphics image processing technology will be more broad.

\section{The Concept of Big Data}

From the literal sense, a large amount of data that is data extremely large data, which is not only a huge amount of information of limited data, including complex data information generating repeat of diversification and data information. With the data of fast and continuous development of contemporary virtual social, big data is the inevitable product of modern society, compared to traditional computing, big data has many advantages, such as large data resource utilization has low cost, high ratio, size, speed and other characteristics. In the production and life, big data can put all the activity data for record keeping, which is its core. Classification analysis, genetic algorithms, machine learning, genetic algorithms and other key technologies are big data.

Big Data with an advanced decision-making ability and insight, compared with traditional data 
software has its elusive data processing capabilities meet the needs of networking and computer development, to a great extent on the social development and people's living habits transformative resistance of. "Wall Street Journal" that big data and intelligent production, Wi-Fi revolution and called to lead the future prosperity of the big technological change. In the era of big data for user groups in terms of the "cloud" is familiar to many systems, the influx of a variety of IT cloud disk, cloud photos, music and other cloud provides virtually unlimited resources of the Internet, the use of virtual data, easy for users to share anywhere in the cloud resources, from the earliest computers to the popularity of flat-panel and mobile phone, only the network and big data "clouds", you can say goodbye before the original operation.

\section{The Challenges of the Big Data Era}

Data mining claim becomes high. Mainly reflected in the data collected comprehensiveness and credibility. Require visualization of the results. Great attention data is relatively high, the potential of the data is relatively large and it is easier to become a target for hackers. It will gather a lot of data is greater this loss. At the same time, large amounts of data aggregation and centralized storage, increasing the risk of data leakage, because there is no definition of ownership and use of data, big data will involve a lot of individual privacy. In addition, the consequences of a large data set are stored with a lot of complex data, security means and measures demanding. Big Data technologies has its advantages, hackers could exploit big data technology to attack, resulting in protection of information security more difficult. The era of big data requires specialized personnel. Big Data technology is a new technology, personnel training requires a certain period and the market needs a lot of innovative talents of big data, major talent gap, Chinese talent on big data applications and even fewer.

\section{The Overview on Computer Graphics Processing Technical Innovation}

Computer image processing technology is the geometric model and descriptive data of the image or concept of the software in a computer system to store, finalized, optimize, modify and show. Computer graphics and image processing techniques can be used to design graphic colors, textures and shading to make the texture processing for image modeling design and modeling, image eliminate hidden lines and hidden surfaces, curves and surfaces for the graphic to fit the operation, digitization image storage, image segmentation, analysis, coding, enhancement, recovery and other operations, as well as the form of the image conversion, such as projection, scaling, rotation, translation, and other geometric forms.

Computer hardware performance is good or bad for the computer graphics image processing effects have a direct impact on computer graphics image processing software of the terminal display and computer together, because the computer image processing technology has its own design, store, modify other functions, can rapid integration of image data, not only can protect the treatment effect of computer graphics, but also can effectively improve the computer's central processor operating results and computer graphics image processing software. Keyboard and mouse as the input device terminal, can be completed on the graphics changes and positioning, and the use of monitors, plotters, printers and other display devices and output devices, you can save the entire computer image.

Computer image processing technology mainly has five basic functions: the dialogue, input, output, storage and computing. Conversations feature is the use of interactive communications equipment and computer monitors to achieve human-machine communication. Input and output function refers to a computer graphics image processing software can always inputs and outputs associated graphic images. Memory function refers to the number of real-time monitoring computer graphic image data for effective retrieval and maintenance. Computing refers to a computer graphics image processing software for graphic images related to the necessary exchange of data and computational analysis.

Hardware configuration of the computer image processing technology, including workstations 
and microcomputers, software configuration is built on the workstation and microcomputer running software. Computer image processing technology workstation software has TDI and Alias are two types of workstation software is mainly responsible for dealing with computer workstations in various image processing. Computer graphics, image processing software on a microcomputer including 3DStudio, morph and Photoshop, 3DStudio is a leading image processing software on a microcomputer, is widely used in many computer systems; morph is a commonly used two-dimensional graphic image processing software, can be made into a graphic image or another graphic or image; Photoshop is a very professional image processing software, which supports the separation plate information of a graphic image, graphic image to the people to processing bring a lot of convenience.

\section{The Computer Graphics Processing Technology in the Big Data Era}

Distributed Data Storage. Distributed data processing technology implementation by the Google company's proposed GFS technology. This technology in IBM, Baidu and other companies get a lot of use and rapid development. Distributed storage utilization concept column stores. Column is stored as a unit for storage, compared to the line memory having data compression, fast cycling and so on. Today the ranks of the more popular hybrid storage architecture technology, the structure can quickly load large amounts of data query time is shortened, and efficient use of disk space. In the study, to continue to optimize the layout of the data storage method of distribution. Improve the efficiency of large storage and processing of data.

Efficient Indexing of Data. Google company's proposed BIGTABLE technology is the mainstream technology index. The current research focus is clustered index and complementary clustered index. Wherein while a clustered index is stored in the order according to index all of the data structure. The complementary clustered index is created using multiple copies of complementary column index table for the index. Combined with the results of the query method used to estimate, plan optimal data query.

The Content Information Based on Data Mining. Based on the content of the main content of the data mining technology for Web search and related entities. Today's hot Internet information search elements to rank learning algorithm, sorting algorithm is proposed mainly for learning information, the characteristics of social media attention of social media data for short text features, sorting feature learning algorithm is based on this proposed common Sort learning algorithm main point by point, the pair-wise and column by column.

Genetic Algorithms and Neural Networks. Proposed genetic algorithm is a random search approach to learn the law of evolution of biological evolution, the genetic approach using probabilistic optimization approach, which can automatically adjust the search direction. Genetic algorithms have been applied in technology, machine learning, signal processing, logistics and other aspects of the site. Proposed neural network inspired by biological neural network structure and functioning. Neural network algorithm Neural networks simulate the behavior of the animal movement, it is distributed parallel information processing of mathematical algorithms.

\section{The Application of Computer Graphics Processing Technology}

User Interface. People using a computer system user interface to operate a variety of computer software, computer graphics and image processing technology and user interfaces effective combination, by means of a computer operating system to build friendly interactive graphical user interface, which greatly improved the image processing computer graphics simplicity and ease of use. In recent years, Microsoft popularize and promote the image of the windows system, give full play to the important role of computer graphics processing technology and comprehensive user interface integration.

Animation and Art. With the rapid development of computer science and technology, computer hardware and computer graphics is also booming, static graphic image has been difficult to meet people's huge demand for high-quality, high-quality, dynamic graphic images, so in recent years, 
computer animation technology to flourish, especially in some art designers, mostly rely on computer graphics and image processing software for artistic creation. The rapid development of computer graphics and image processing technology, while promoting the use and development of art and design technology, for example, 3DS Studio Max dimensional three-dimensional design software and Photoshop graphic design software.

Scientific Visualization. In recent years, the rapid development of China's socialist market economy, information and communication in various fields more and more frequent, more widespread use and popularity of computer network technology, enable the computer system increasingly large amount of information in a database, computer data processing and analysis techniques facing serious test. Related technical operations staff use computer data processing and analysis software, is difficult to accurately and quickly loading equipment program, this one is not required to intervene, and about half were more than a minute, the next box will pop up some information you need to manually input, this process is very simple and only need to press the tips of the steps can be, but also to wait until some time after the last restart, OK, this installation of Windows XP so you're done. In this it is worth reminding, remember to Windows XP serial number on a piece of paper to record in advance, because it was used during the installation manual input to enter the next step, of course, if you are to get it 25 Remember the whole string, which is a different matter.

\section{Conclusions}

Big Data era for computer information processing technology is both an opportunity and a challenge, more and more people will use computer technology to analyze the data, which will make the computer information processing technology is more widely used, but also for computer information processing technology put forward higher requirements, need to constantly improve there are still insufficient, and based on massive data processing needs to adapt to a truly big data era of network systems and security systems, computer information processing technology to wider areas.

\section{References}

[1] Han Xiaoying. Fujian Computer, Vol. 6 (2004) No 53, p.25-26

[2] Wang Yunhui, Wang Qunyong. Information and Computer, Vol. 12 (2005) No 27, p.74-76

[3] Jing Jianfen, Hou XuSiem. Tianjin Institute of Technology, Vol. 30 (2004) No 19, p.144-145

[4] Wang Kuailiang. World Scientific, Vol. 29 (2008) No 27, p.21-23

[5] Zhang Gongxu. Heilongjiang Science and Technology Information, Vol. 4 (2011) No 27, p. $281-289$ 